\title{
Vehicle Routing Problem with Simultaneous Pickup and Delivery
}

\author{
San Nah Sze ${ }^{\mathrm{a}, 1}$, Siaw Ying Doreen Sek ${ }^{\mathrm{a}, 2}$, Jeeu Fong Sze ${ }^{\mathrm{a}, 3}$, Wai Shiang Cheah ${ }^{\mathrm{a}, 4}$, Kang Leng Chiew ${ }^{\mathrm{a}, 5}$ \\ ${ }^{a}$ Faculty of Computer Science and Information Technology, Universiti Malaysia Sarawak, Kota Samarahan, 94300, Sarawak, Malaysia, \\ E-mail: ${ }^{1}$ snsze unimas.my; ${ }^{2}$ doreenying92@gmail.com; ${ }^{3}$ jfsze unimas.my; ${ }^{4}$ wscheah; ${ }^{5}$ klchiew@unimas.my
}

\begin{abstract}
This paper focuses on the Vehicle Routing Problem with Simultaneous Pickup and Delivery (VRPSPD) issue. VRPSPD is one of the extended problems related to the usual Vehicle Routing Problem (VRP). VRPSPD consists of both linehaul customers and backhaul customers with known demand. In VPRSPD, only a single depot can receive and supply the loads. The vehicles can only visit each customer once and can serve all customers simultaneously by delivering or picking up the loads within a limited capacity. VPRSPD is an NP-hard problem. The considerable data size has increased the difficulty for it to be solved by using mathematical programming or combinatorial optimization. A heuristic approach based on the Variable Neighborhood Search (VNS) is proposed. Heuristic-based solutions offer feasible solutions that are approximately accurate to the exact solution. It is one of the most popular solutions to solve a complex problem. In this research, the algorithm solution consists of two main phases. A simple heuristic is used to generate the initial solution in the first phase. Then, the feasible solution obtained in the first phase will become the initial input for the improvement phase, which applied the VNS algorithm. The proposed algorithm is tested using a list of benchmark datasets. The result obtained is compared with the best solution that can be found in literature research. The comparison result shows that the heuristic algorithm is favorable to be used for this kind of vehicle routing problem.
\end{abstract}

Keywords — vehicle routing problem; heuristic algorithm; delivery pickup customers; variable neighborhood search.

\section{INTRODUCTION}

The Vehicle Routing Problem (VRP) is a significant logistics problem with a wide range of applicability. VRP aims to come up with suitable routes for a fleet of homogeneous vehicles that can serve a group of customers that start and end at a depot by minimizing the traveling distance. Each customer can only be served once by one vehicle at a time. VRP was first introduced by Dantzig and Ramser [1] in the research, which targets to provide an optimum routing solution with minimum travel distance between a fleet of gasoline delivery trucks and a list of terminals with various service stations supplied. In the year 1964, Clarke and Wright [2] introduced a new variant of VRP that will commonly occur in logistics and transport fields, such as considerably more than one vehicle in the problem formulation, mode to serve the customer or vehicle with varying capacities. This is since the source of the problem is attributed to the numerous modes used to serve the customer and the varying capacities handled by more than one vehicle.

On that note, research was commenced to apply or combine VRP with different constraints and parameters. VRP is one of the most popular research topics. For example, VRP with time window [3], VRP with a capacity [4], VRP with multiple depots [5], VRP with pickup and delivery [6], and vehicle routing with split deliveries [7]. Green vehicle routing [8] are some of the joint research topics related to regular VRP that were conducted in the past few years. During the last few decades, VRP had begun to be applied in solving various kinds of real-life logistics problems. Different methodologies and techniques were investigated to solve the VRP problem. There are a considerable number of methods and solutions that can be found in published academic literature.

Furthermore, the development of VRP software and applications displayed rapid growth in the industry and logistics fields. Thousands of companies have started to implement this technology into the logistics management process, such as the support system used by an oil downstream company, which was proposed by Gayialis and Tatsiopoulos in the year 2004 [9]. Likewise, the scheduling model was used by a sales company for flight tickets to arranging their free pickup and delivery services [10]. Moreover, literature research has proven that the optimized routing solution reduces transportation cost [11], amount of resources required, fuel consumption [12], and the emissions of greenhouse gas, such as carbon dioxide, especially in urban areas [13], [14].

Vehicle Routing Problem with Simultaneous Pickups and Deliveries (VRPSPD) is one of the additional problems that stem from the usual VRP. VRPSPD consists of both linehaul customers and backhaul customers with known demand. According to Pinto, Alves, and Carvalho [15], linehaul 